\title{
Application of the Peierls-Nabarro Model to Symmetric Tilt Low-Angle Grain Boundary with Full <a> Dislocation in Pure Magnesium
}

\author{
Tou-Wen Fan $^{1} \cdot$ Xiu-Bo Yang ${ }^{1,2} \cdot$ Jiang-Hua Chen ${ }^{1,3} \cdot$ Ling-Hong Liu ${ }^{1,4} \cdot$ Ding-Wan Yuan ${ }^{1} \cdot$ Yong Zhang $^{1} \cdot$ \\ Cui-Lan Wu ${ }^{1}$
}

Received: 10 May 2016/Revised: 26 June 2016/Published online: 7 September 2016

(c) The Chinese Society for Metals and Springer-Verlag Berlin Heidelberg 2016

\begin{abstract}
Three types of symmetric (1120) tilt low-angle grain boundaries (LAGBs) with array of basal, prismatic, and pyramidal edge full $<\mathrm{a}>$ dislocations in pure $\mathrm{Mg}$ have been studied by using the improved Peierls-Nabarro model in combination with the generalized stacking fault energy curve. The results show that with decreasing distance between the dislocations in all the three types of tilt LAGBs, the stress and strain fields are gradually suppressed. The reduction extent of the stress and strain fields decreases from the prismatic to basal to pyramidal dislocations. The variation of dislocation line energy (DLE) for all tilt LAGBs is divided into three stages: DLE changes slightly and linearly when the distance is larger than $300 \AA, \sim 10 \%$; DLE declines exponentially and quickly when the distance goes from 300 to $100 \AA$, $\sim 70 \%$; and finally, the descent speed lowers when the distance is smaller than $100 \AA$ and the dislocation core energy is nearly half of the DLE. The grain boundary energy (GBE) decreases when the tilt angle of LAGB increases from $1^{\circ}$ to $2^{\circ}$ for all cases. The tilt LAGB consists of pyramidal dislocations always has the largest GBE, while that with array of prismatic dislocations has the smallest one in the whole range. The Peierls stress of dislocation in tilt LAGB is nearly unchanged, the same as that of single dislocation. This work is useful for further study of dissociated dislocation, solute segregation, precipitate nucleation in tilt LAGB and its interaction with single dislocations.
\end{abstract}

KEY WORDS: Tilt grain boundary; Generalized stacking fault energy; Magnesium alloy; Dislocation; Peierls stress

Available online at http://link.springer.com/journal/40195

Tou-Wen Fan

fantouwen@hnu.edu.cn

Xiu-Bo Yang

yangxiubo4979@yeah.net

1 Center for High-Resolution Electron Microscopy, College of Materials Science and Engineering, Hunan University, Changsha 410082, China

2 Department of Applied Physics, School of Physics and Electronics, Hunan University, Changsha 410082, China

3 Hunan Province Key Laboratory for Spray Deposition Technology and Application, Hunan University, Changsha 410082, China

4 College of Science, Central South University of Forestry and Technology, Changsha 410004, China

\section{Introduction}

Magnesium alloys hold promising future in electronic, automotive, aerospace, and biomedical applications due to their lightweight, high specific strength, damping properties, and recyclability $[1,2]$. However, the poor formability and limited ductility at room temperature originated from the hexagonal (hcp) crystal structure (except for some special Li-containing alloys) and the consequent nonavailability of adequate numbers of independent slip systems still severely limit the applications of the alloys [3-5]. The inherent difficulty can be reasonably overcome by some special processing of severe plastic deformation (SPD), such as equal-channel angular press (ECAP) [6-16], accumulative rolling bounding (ARB) [17-19], high pressure torsion (HPT) [20], and continuous variable 
cross-section direct extrusion (CVCDE) [21]. High strength or good ductility, even superplasticity, in aluminum [22-24], copper [25-27], and magnesium alloys [12, 28-31] has been obtained owing to the achieved ultrafine grain (UFG) during these processes. Valiev et al. $[32,33]$ pointed out that the unusual stress-strain curves of $\mathrm{Cu}$ and Ti might be explained in terms of the change in the deformation mechanism from the dislocation slip/twinning to grain boundary sliding (GBS) in SPD metals. Zhao et al. [34] demonstrated that a large fraction of high-angle grain boundaries (HAGBs) and a low dislocation density may improve significantly the toughness and uniform elongation of UFG Cu. Biswas et al. [35] found that high proportion of low-angle grain boundaries (LAGBs) also presented in ECAPed Mg alloys. The LAGBs may transform to HAGBs through attracting dislocations $[36,37]$ and thus reduce the dislocation density. Therefore, LAGBs have a strong effect on the strength and ductility of UFG Mg alloys. Molnár et al. [38] discovered recently that the strain gradient around LAGBs affects significantly the generation of UFGs of $\mathrm{Mg}$ alloys during the ECAP. However, up to now, the theoretical investigation of LAGBs of $\mathrm{Mg}$ alloys is very scarce owing to the difficulties in treatment of the dislocation cores, and the strain and stress states of LAGBs are still unknown. Therefore, it is necessary and valuable to study LAGBs in pure Mg firstly.

It is generally accepted that symmetric tilt LAGBs can be successfully described in terms of dislocation arrays [39-42]. Before the advent of high-resolution transmission electron microscopy (HRTEM) and atomistic simulation, LAGBs are usually treated by Read-Shockley model [43]. This approach succeeded because LAGB was elastically distorted in most regions. These models, however, were incomplete, because they did not contain such effects as stacking faults or included a complicated treatment of the dislocation cores. Another way to deal with LAGB is the direct molecular dynamics (MD) simulations based on empirical potential, such as Lennard-Jones (LJ) potential [44] and embedded atomic method (EAM) [45-47]. However, it is difficult to construct a big supercell to simulate dislocations, and the results often show large difference [48], e.g., the Peierls stresses at $T=0 \mathrm{~K}$ for basal slip are scattered from a few mega-Pascals by using Sun potential [45] to ten times larger by using Liu potential [46], depending on the fitting parameters to the predetermined database. Also, to date, this approach is still limited to pure metals due to the difficulty in fitting the potentials of alloys [49]. An alternative method is the one based on the framework of Peierls-Nabarro $(\mathrm{P}-\mathrm{N})$ model. The simple and effective $\mathrm{P}-\mathrm{N}$ model has been successfully applied to describe the dislocation core or twinning properties of metals, intermetallics, solid solutions, and compounds [50-61] by combining the generalized stacking fault energy (GSFE or $\gamma$ ) surface obtained from accurate first-principles calculations by sliding one-half of a crystal over the other half [62]. Dai et al. [63, 64] have recently used the generalized $\mathrm{P}-\mathrm{N}$ model to study the structure and energy of (111) twist boundaries in face-centered cubic (fcc) $\mathrm{Al}, \mathrm{Cu}$ and $\mathrm{Ni}$ and obtained good results. However, to our knowledge, the dislocation behavior especially the core properties in symmetric tilt LAGB has not been reported. The only one step needed to extend $\mathrm{P}-\mathrm{N}$ model for the investigation of symmetric tilt LAGB is to derive an appropriate formula for the stress field. By minimizing the restoring force derived from GSFE or $\gamma$ curve and stress field, the detailed parameters can be obtained, and the properties of symmetric tilt LAGB can be studied. It is conceivable that this method is useful for further study of tilt LAGBs in other metals and alloys.

Although the full $<\mathrm{a}>$ dislocation on (0001) basal plane of $\mathrm{Mg}$ can dissociate into two Shockley partial dislocations, the importance of the LAGBs with array of basal full $<a>$ dislocations can be validated from recent high-angle annular dark-field scanning transmission electron microscopy (HAADF-STEM) experimental investigation about a $\mathrm{Mg}_{97} \mathrm{Zn}_{1} \mathrm{Y}_{2}$ (at.\%) alloy, where Cottrell atmospheres along full $<\mathrm{a}>$ dislocations in LAGBs of long-period stacking ordered (LPSO) phases have clearly been observed at atomic scale resolution [65]. This work aims to investigate three types of symmetric (1120) tilt LAGBs with array of basal, prismatic, and pyramidal edge full $<\mathrm{a}>$ dislocations, respectively, in pure $\mathrm{Mg}$ by using the combination of the generalized $\mathrm{P}-\mathrm{N}$ model with the GSFE surface. The structures of all the three types of LAGBs are presented in Fig. 1. The theoretical model and computational methods are provided in Sect. 2. In Sect. 3, the stress field and disregistry profiles for various symmetric tilt LAGBs have been obtained first; then the dislocation line energy (DLE) as a function of distance between dislocations and the corresponding grain boundary energy (GBE) against tilt

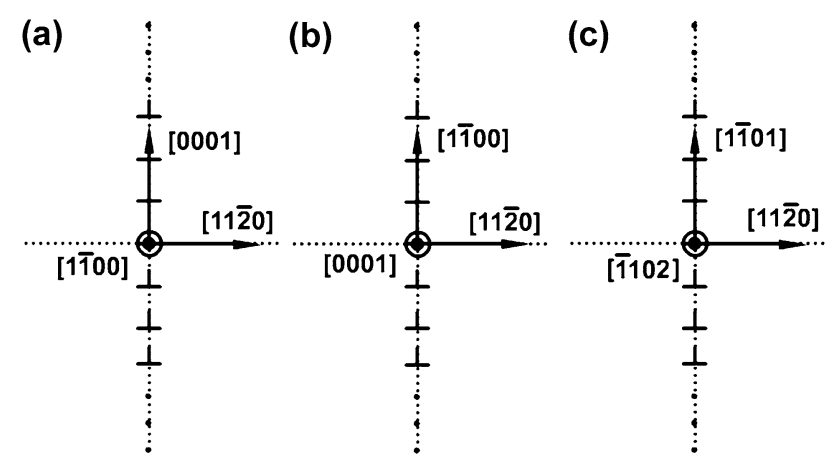

Fig. 1 Illustration of symmetric (1120) tilt low-angle grain boundary with array of (0001) basal a, (1100) prismatic b, (1101) pyramidal c full edge $<\mathrm{a}>$ dislocations, respectively 
degree between grains are gained. Finally, the motion characters of LAGBs are investigated through calculating the Peierls stress. A summary is provided at the end of the paper.

\section{Computational Methods and Theoretical Model}

\subsection{Computational Methods}

The calculations of GSFE surface were done using the Vienna ab initio simulation package (VASP) [66-68] based on density functional theory (DFT). The projector-augmented wave (PAW) [69] potential was used to treat corevalence interaction, and the Perdew-Burke-Ernzerhof (PBE) [70] version of generalized gradient approximation (GGA) was employed to describe the exchange-correlation functional. In all calculations, the cutoff energy of plane wave basis was taken as $500 \mathrm{eV}$. Brillouin zone sampling was performed using Gamma-centered Monkhorst-Pack grids [71]. The $1 \times 1 \times 12,1 \times 1 \times 16$ and $1 \times 1 \times 18$ supercells with a vacuum region of about $10 \AA$ between the periodically repeated slabs were adopted for basal

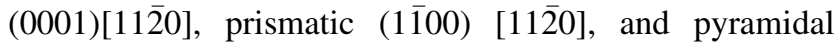

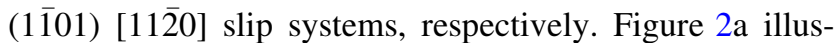
trates the models used to calculate the GSFE of pure $\mathrm{Mg}$ for various slip systems, where arrows stand for the crystal directions. During simulations, all the atoms were fully relaxed using a quasi-Newton algorithm until the Hellmann-Feynman force is reduced within $0.02 \mathrm{eV} / \AA$, except that atoms in the two planes of relative slippage were only allowed to relax in the direction perpendicular to glide plane. After adequate tests, $15 \times 9 \times 1$ k-point meshes for all supercells were utilized. The convergence tests with respect to these parameters show that the error bar for the total energy is less than $1 \mathrm{meV} / \mathrm{atom}$.

The GSFE is defined as the difference between the total energies of two supercells constructed to simulate faults with $u=0$ and $u \neq 0$ [72] and is calculated according to the following expression:

$\gamma_{\mathrm{GSF}}(u)=\frac{E(u)-E(0)}{A}$.

where $E(u)$ and $E(0)$ are the total energies of the supercells with and without the fault vector $u$, respectively, and $A$ denotes the area of the fault plane. For the investigation of dislocations, the GSFE curve is expanded in Fourier series in reciprocal lattice vectors:

$$
\begin{aligned}
\gamma_{\mathrm{GSF}}(u)= & c_{0}+c_{1} \cos \left(\frac{2 \pi u}{a}\right)+c_{2} \cos \left(\frac{4 \pi u}{a}\right) \\
& +c_{3} \cos \left(\frac{6 \pi u}{a}\right)+c_{4} \cos \left(\frac{8 \pi u}{a}\right),
\end{aligned}
$$

where $a=3.21 \AA$ is the lattice constant of $\mathrm{Mg}$ and $c_{0}, c_{1}$, $c_{2}, c_{3}$ and $c_{4}$ are the coefficients.
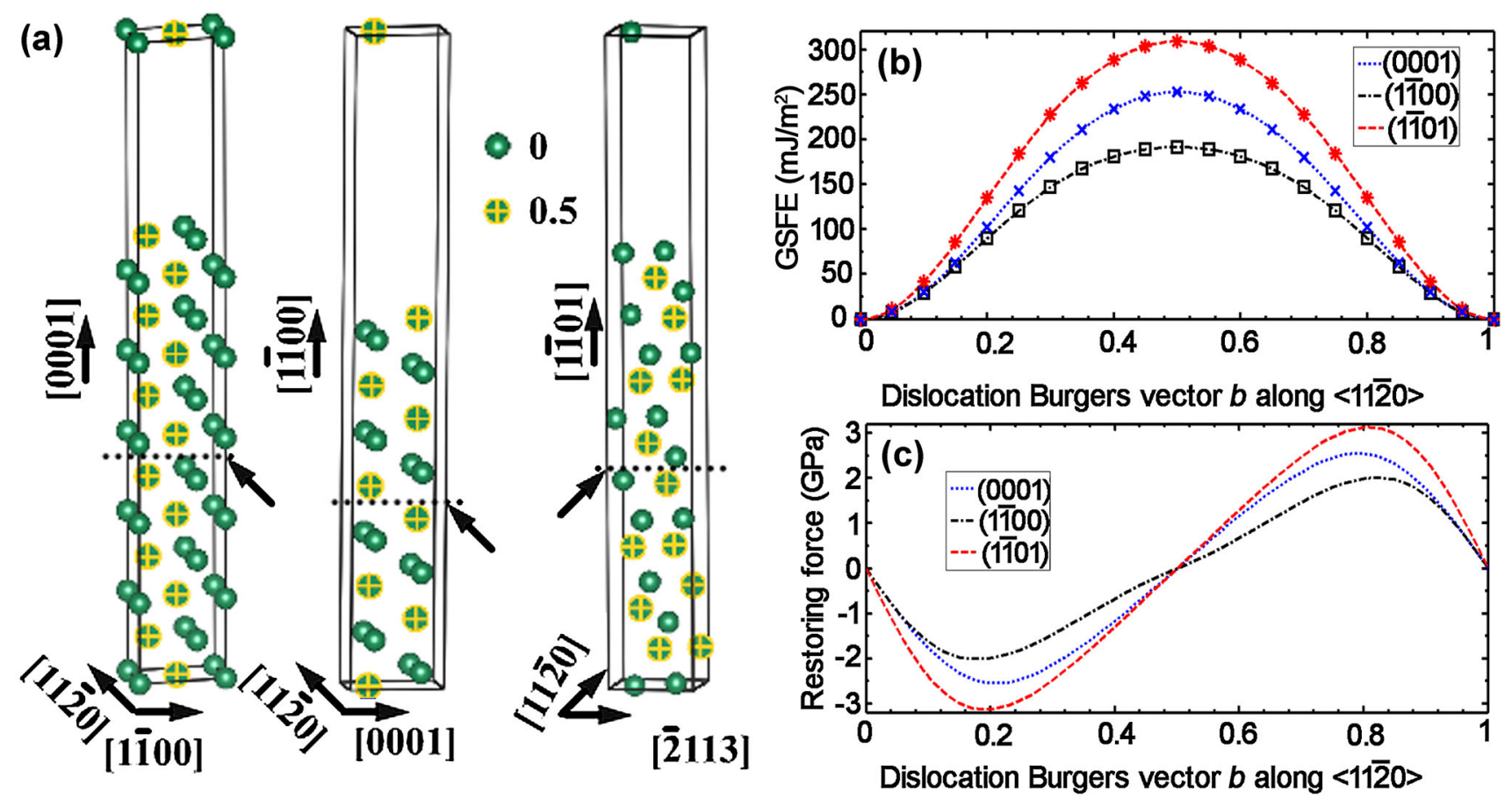

Fig. 2 a Supercells used to calculate the GSF energy of (0001) basal, (1100) prismatic, and (1101) pyramidal planes in pure Mg, 0 and 0.5 representing different positions along the direction perpendicular to paper. An about $10 \AA$ thick vacuum is added. b Corresponding GSF energies and $\mathbf{c}$ restoring forces 


\subsection{Theoretical Models}

According to Peierls-Nabarro model of a dislocation, the shear stress along the slip plane for edge dislocation [41] is:

$$
\begin{aligned}
\sigma_{x y}= & \frac{-K b}{2 \pi}\left[\frac{x}{x^{2}+(y+\zeta)^{2}}-\frac{2 x y(y+\zeta)}{\left[x^{2}+(y+\zeta)^{2}\right]^{2}}\right] \\
= & \frac{-K b}{2 \pi}\left[\frac{x}{x^{2}+(y+\zeta)^{2}}-\frac{2 x(y+\zeta)^{2}}{\left[x^{2}+(y+\zeta)^{2}\right]^{2}},\right. \\
& \left.+\frac{2 x \zeta(y+\zeta)}{\left[x^{2}+(y+\zeta)^{2}\right]^{2}}\right]
\end{aligned}
$$

where $x$ is the orientation perpendicular to the dislocation line on the slip plane, $y$ is the normal direction of the slip plane, and $z$ is the axis along the dislocation line. $K, b$ and $\zeta$ represent the energy factor, burgers vector, and half width of dislocation, respectively. In the region $y<0$, the factor $\zeta$ becomes $-\zeta$.

Summing the contributions of the individual dislocation in symmetric tilt LAGB, one finds:

$$
\begin{aligned}
\sigma_{x y}= & \frac{-K b}{2 \pi} \sum_{n=-\infty}^{+\infty}\left[\frac{x}{x^{2}+(y+n D+\zeta)^{2}} .\right. \\
& \left.-\frac{2 x(y+n D+\zeta)^{2}}{\left[x^{2}+(y+n D+\zeta)^{2}\right]^{2}}+\frac{2 x \zeta(y+n D+\zeta)}{\left[x^{2}+(y+n D+\zeta)^{2}\right]^{2}}\right] \\
= & \frac{-K b}{2 \pi} \sum_{n=-\infty}^{+\infty}\left[\frac{X / D}{X^{2}+(n+Y)^{2}}-\frac{2 X(n+Y)^{2} / D}{\left[X^{2}+(n+Y)^{2}\right]^{2}}\right. \\
& \left.+\frac{2 X \zeta(n+Y) / D^{2}}{\left[X^{2}+(n+Y)^{2}\right]^{2}}\right]
\end{aligned}
$$

where $D$ represents the mean distance between dislocations in the tilt boundary. $X=\frac{x}{D}$ and $Y=\frac{y+\zeta}{D}$. The basic formula used in the sum is

$$
\begin{aligned}
& \sum_{n=-\infty}^{+\infty}\left[\frac{n+p}{q^{2}+(n+p)^{2}}\right]=\frac{\pi \sin 2 \pi p}{\cosh 2 \pi q-\cos 2 \pi p}, \\
& \sum_{n=-\infty}^{+\infty}\left[\frac{1}{q^{2}+(n+p)^{2}}\right]=\frac{\pi}{q} \frac{\sinh 2 \pi q}{\cosh 2 \pi q-\cos 2 \pi p} .
\end{aligned}
$$

Differentiating Eqs. (5a) and (5b) with respect to $q$ and $p$, respectively, one obtains,

$$
\begin{aligned}
& \sum_{n=-\infty}^{+\infty} \frac{n+p}{\left[q^{2}+(n+p)^{2}\right]^{2}}=\frac{\pi^{2}}{q} \frac{\sinh 2 \pi q \sin 2 \pi p}{(\cosh 2 \pi q-\cos 2 \pi p)^{2}} \\
& \sum_{n=-\infty}^{\infty} \frac{-(n+p)^{2}}{\left[q^{2}+(n+p)^{2}\right]^{2}}=\frac{\pi^{2}(\cosh 2 \pi q \cos 2 \pi p-1)}{(\cosh 2 \pi q-\cos 2 \pi p)^{2}} \\
& -\frac{\pi \sinh 2 \pi q}{2 q(\cosh 2 \pi q-\cos 2 \pi p)}
\end{aligned}
$$

According to above formulas, Eq. (3) can be simplified as:

$$
\sigma_{x y}=\frac{\pi K b}{D^{2}} \frac{X D(\cosh 2 \pi X \cos 2 \pi Y-1)+\zeta \sinh 2 \pi X \sin 2 \pi Y}{(\cosh 2 \pi X-\cos 2 \pi Y)^{2}} .
$$

As for the sign changing while $y<0$, it is found that this has little effect in simulation processing of tilt LAGB by using Eq. (7). Only the peak of shear stress is overvalued. So, to maintain the sign of $\zeta$ across $y=0, \zeta$ is changed into $\lambda \zeta$, where $\lambda=0.975$ is employed for all cases after adequate tests.

When the stress field of dislocation in the LAGB is obtained, the Peierls-Nabarro model can be expanded to solve its structure characterization and mechanical properties. Also, like the Peierls-Nabarro model for single dislocation [41], the misfit region of inelastic displacement is assumed to be restricted to the glide plane, but the linear elasticity applies far from it. A dislocation in the tilt LAGB is treated as a continuous distribution of infinitesimal dislocations $u(x)$ (or disregistry function) with dislocation density $\rho(x)$ condition $\int_{-\infty}^{+\infty} \rho(x) \mathrm{d} x=\int_{-\infty}^{+\infty} \frac{\mathrm{d} u(x)}{\mathrm{d} x} \mathrm{~d} x=b$ along the glide plane. The restoring stress $\sigma_{x y}^{\prime}(u)$ acting between atoms on either sides of the interface is balanced by resultant stress of the sum in Eq. (7). As largely developed in numerous studies since Vitek [62], the solution of the $\mathrm{P}-\mathrm{N}$ model for tilt LAGB can be numerically found by introducing a restoring stress simply defined as the gradient of the so-called GSFE $\gamma$,

$\sigma_{x y}^{\prime}(u)=-\frac{\partial \gamma_{\mathrm{GSF}}}{\partial u}$

The disregistry $u(x)$ of dislocations in tilt LAGB was considered to have the same trial function as that of single dislocation,

$u(x)=\frac{b}{2}+\frac{b}{\pi} \sum_{i=1}^{N} \alpha_{i} \arctan \frac{x-x_{i}}{\zeta_{i}}$

where $\alpha_{i}, x_{i}$, and $\zeta_{i}$ are variational constants. By taking the above form of $u(x)$, the infinitesimal dislocation density $\rho(x)$ can be expressed as: 
$\rho(x)=\frac{\mathrm{d} u(x)}{\mathrm{d} x}=\frac{b}{\pi} \sum_{i=1}^{N} \alpha_{i} \frac{\zeta_{i}}{\left(x-x_{i}\right)^{2}+\zeta_{i}^{2}}$.

Since the disregistry $u(x)$ and the dislocation density $\rho(x)$ must be solutions of the normalization condition, $\alpha_{i}$ is constrained by $\sum_{i=1}^{N} \alpha_{i}=1$. Based on above trial solution of disregistry, Eq. (7) can be revised accordingly:

\section{Results and Discussion}

The GSFE curves and the corresponding restoring forces have been firstly obtained, and the results are shown in Fig. $2 b$ and c, respectively. Typical values of maximum GSFE with other theoretical predictions are listed in Table 1. It is seen that the values are in general agreement

$\sigma_{x y}=\sum_{i=1}^{N} \alpha_{i} \frac{\pi K b}{D} \frac{\left(X-X_{i}\right)\left[\cosh 2 \pi\left(X-X_{i}\right) \cos 2 \pi Y_{i}-1\right]+\lambda \zeta_{i} \sinh 2 \pi\left(X-X_{i}\right) \sin 2 \pi Y_{i}}{\left[\cosh 2 \pi\left(X-X_{i}\right)-\cos 2 \pi Y_{i}\right]^{2}}$,

where $X_{i}=\frac{x_{i}}{D}$ and $Y_{i}=\frac{y+\lambda \zeta_{i}}{D}$. The constants $\alpha_{i}, x_{i}$ and $\zeta_{i}$ are determined by minimizing the expression

$\Delta=\left|\sigma_{x y}\left(\alpha_{i}, \zeta_{i}, x_{i} ; x\right)-\sigma_{x y}^{\prime}\left[u\left(\alpha_{i}, \zeta_{i}, x_{i}\right)\right]\right|$,

$N=5$ is sufficient to provide a satisfactory fit. Another important physical quantity is the mean energy of dislocation in the symmetric tilt LAGB, of which the treatment is similar to the calculation of the energy of Peierls dislocation [41]. The equilibrium configuration of discrete dislocations in the symmetric tilt LAGBs is obtained by setting the stress arising from the misfit of the bonds (mentioned as GSFE surface) in the glide plane equal to the stress of all dislocations in the LAGBs which acts on the surface of a half-crystal. Thus, the mean elastic strain energy of dislocations in symmetric tilt LAGBs equals the work done by surface forces in generating the displacement $u(x)$ [41]. The misfit energy, corresponding to the core energy of the dislocation in the symmetric tilt LAGB, is also a sum of total chemical energy to distort all bonds across the glide plane $[62,73,74]$. Then the mean energy of single dislocation in symmetric tilt LAGBs can be given as the sum of elastic and misfit energy

$E_{\text {total }}=E_{\text {elastic }}+E_{\text {misfit }}=\int_{-R}^{R}\left(\sigma_{x y} u_{x}\right) \mathrm{d} x+\int_{-R}^{R} \gamma_{\mathrm{GSF}} \mathrm{d} x$,

$R=10^{4} \AA$ represents the normal outer cutoff radius of the elastic solution. The Peierls stress can then be calculated from the formula:

$\sigma_{\mathrm{PN}}=\max \left\{-\sum_{n=-\infty}^{\infty} \sigma_{x y}^{\prime}[u(n a+t)] \cdot \rho\right\}$,

where $a$ is the lattice spacing along the burgers vector and $0 \leq t \leq 1$ represents the exact position within the crystal lattice. with previous theoretical calculations [48, 75-80], indicating that the present calculations are reasonable and reliable. From Fig. 2, we find that both the maximum GSFE and maximum restoring force of pyramidal

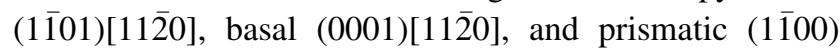
[1120] slip systems are in descending order, being 309, 253, $195 \mathrm{~mJ} / \mathrm{m}^{2}$ and $3.13,2.54,2.01 \mathrm{GPa}$, respectively. The edge full $<\mathrm{a}>$ dislocations on pyramidal (1 $\overline{1} 01)$ plane are difficult to move, while the one on the prismatic (1 $\overline{1} 00)$ plane is the easiest slip system. The fitted coefficients for $\gamma$ surfaces are listed in Table 2. However, in most cases, the dislocations on the (0001) basal plane of $\mathrm{Mg}$ can generally dissociate into two Shockley partial dislocations separated by a distance $(d)$ bound a ribbon of stacking fault. The energy barrier of partials along $<1 \overline{1} 00>$ directions on basal

Table 1 Calculated maximum stacking fault energy $\left(\mathrm{mJ} / \mathrm{m}^{2}\right)$

\begin{tabular}{llll}
\hline & $(0001)$ & $(1 \overline{1} 00)$ & $(1 \overline{101})$ \\
\hline This work (DFT) & 253 & 195 & 309 \\
Pei et al. (DFT) [75] & 276 & 231 & 343 \\
Muzyk et al. (DFT) [76] & 288 & - & - \\
Yasi et al. (DFT) [48] & - & 218 & - \\
Shin et al. (DFT) [77] & - & 230 & 340 \\
Nogaret et al. (DFT) [78] & - & - & 310 \\
Wu et al. (DFT) [79] & 338 & - & - \\
Zu et al. (EAM) [80] & - & - & 210 \\
\hline
\end{tabular}

Table 2 Fitted coefficients $c_{0}, c_{1}, c_{2}, c_{3}$ and $c_{4}$ for basal (0001),

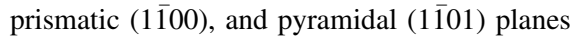

\begin{tabular}{lllllr}
\hline & \multicolumn{1}{c}{$c_{0}$} & \multicolumn{1}{c}{$c_{1}$} & \multicolumn{1}{c}{$c_{2}$} & \multicolumn{1}{c}{$c_{3}$} & \multicolumn{1}{c}{$c_{4}$} \\
\hline$(0001)$ & 134.70 & -126.00 & -8.29 & -0.29 & 0.12 \\
$(1 \overline{1} 00)$ & 108.50 & -94.64 & -12.46 & -1.06 & -0.36 \\
$(1 \overline{1} 01)$ & 169.50 & -153.10 & -14.60 & -1.48 & -0.17 \\
\hline
\end{tabular}


plane is about $92-94 \mathrm{~mJ} / \mathrm{m}^{2}[48,55,76,81,82]$, smaller than that of the prismatic slip system, and thus the dissociated $<\mathrm{a}>$ dislocations on basal plane are the easiest slip systems. The partials are usually mixed dislocations with both edge and screw components, two-dimensional (2D) $\mathrm{P}-\mathrm{N}$ model is necessary to be considered, and the treatment is very complicated [41]. Therefore, the present work focuses only on the full $<\mathrm{a}>$ dislocation on basal plane. The investigation of dissociated symmetric tilt LAGB would be published elsewhere.

The stress field is vital for the further investigation of interaction between intragranular parallel edge dislocations and symmetric tilt LAGB [83, 84], whereas the strain field of dislocations, especially that of core areas in symmetric tilt LAGBs, is important for studying size-interaction of solute atoms with dislocations $[85,86]$ and precipitate nucleation [87]. Typical values of stress and strain fields described by disregistry profile are illustrated in Fig. 3. It can be seen from Fig. 3a-c that for all cases, with the distance increasing from dislocation core $(x=0)$, the stress increases firstly to the maximum value and then decreases gradually to zero, which is a typical variation feature of stress field of dislocation. The maximum stresses of prismatic, basal, and pyramidal edge full $<\mathrm{a}>$ dislocations in (1120) tilt LAGBs are in ascending order and are not changed with the variation of the distance between the dislocations. With decreasing mean distance between the dislocations or with increasing grain boundary angle, the stress field is gradually suppressed and the stress gradient increases. Thus, the interaction of intragranular parallel edge dislocation with such a grain boundary would also be increased. It is inferred that the high-angle grain boundary (HAGB) would show larger strengthening effect for impeding the movement of dislocations, which is in agreement with experiments [34]. Furthermore, the smaller the maximum GSFE is, the more quickly the stress field changes. The stress field of the prismatic edge $<$ a $>$ dislocation in tilt LAGBs possesses the largest variation with decreasing mean distance between the dislocations, followed by those of basal and pyramidal edge full $<\mathrm{a}>$
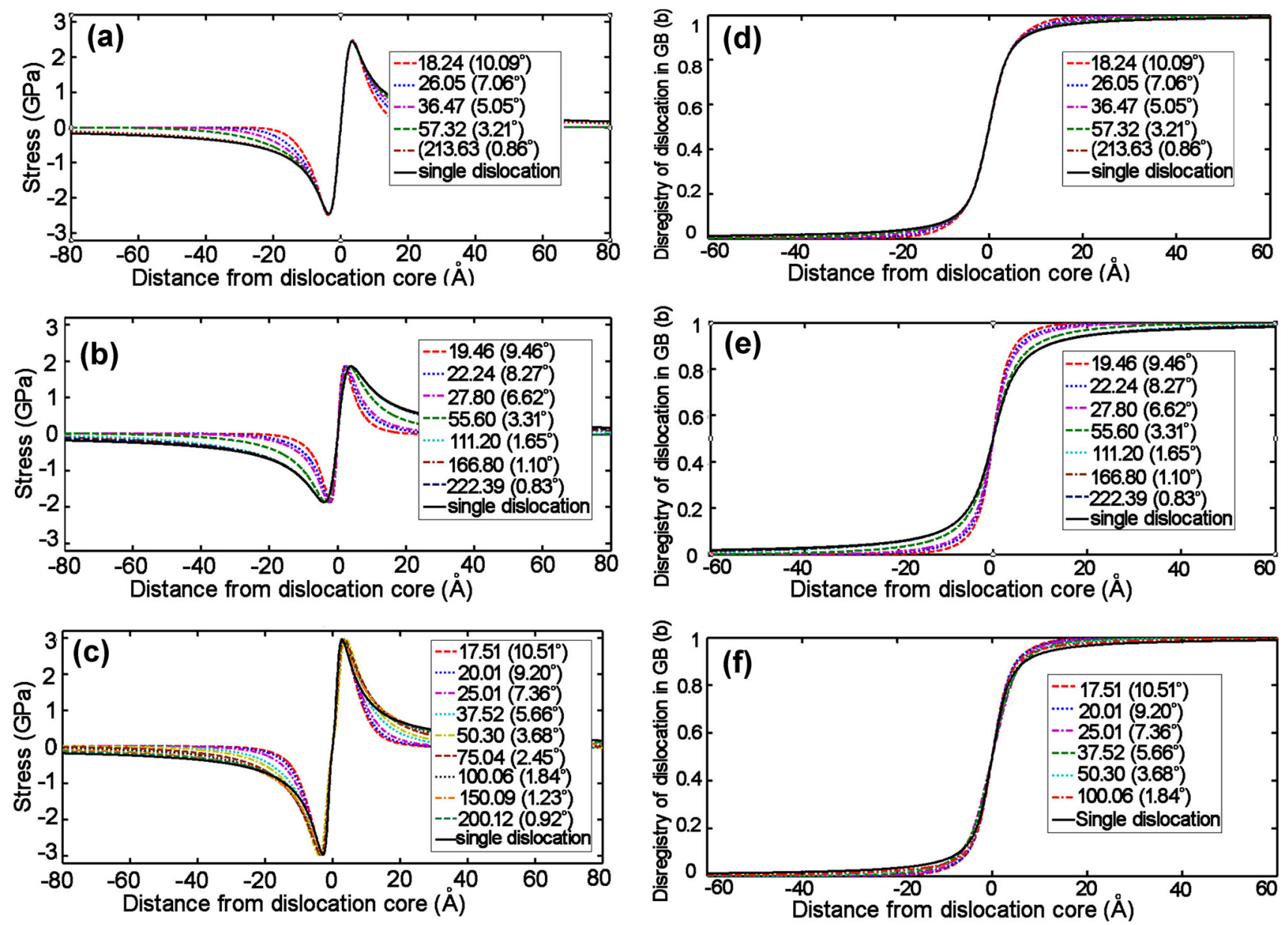

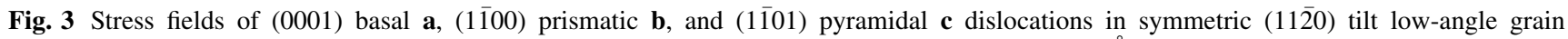
boundaries of $\mathrm{Mg}$, and the corresponding disregistry profiles $\mathbf{d}-\mathbf{f}$, respectively. The mean distance (in $\AA$ ) between dislocations in LAGB and the corresponding tilt angle are included in the inlets 
Table 3 Energy factor $K$, half width $\zeta$, Peierls stress $\sigma_{\mathrm{PN}}$, and the estimated dislocation line energy $E_{\mathrm{dis}}$ and core energy $W_{a^{\prime} / 2}$ obtained from the Peierls-Nabarro model for edge dislocations on the (0001),

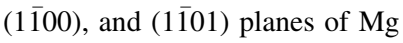

\begin{tabular}{lccc}
\hline & $(0001)$ & $(1 \overline{1} 00)$ & $(1 \overline{1} 01)$ \\
\hline Present $E_{\text {dis }}(\mathrm{eV} / \AA)$ & 1.18331 & 1.16669 & 1.25359 \\
$E_{\mathrm{dis}}(\mathrm{eV} / \AA)$ & 0.92221 & 0.91443 & 0.98658 \\
$K(\mathrm{GPa})$ & 26.34 & 27.32 & 27.65 \\
$\zeta(\AA)$ & 5.39 & 7.28 & 4.73 \\
$\sigma_{\mathrm{PN}}(\mathrm{MPa})$ & 36.09 & 13.71 & 116.52 \\
$W_{a^{\prime} / 2}(\mathrm{meV} / \AA)$ & 134.28 & 140.87 & 136.35 \\
\hline
\end{tabular}

dislocations. After close inspection of the dislocation properties on the three slip planes, it is found that the prismatic edge $<\mathrm{a}>$ dislocation has a much larger half width (see Table 3) than the other two, which would result in more atoms contained at the strong interaction area, and thus both the strain and stress fields change faster. As the distance between dislocations decreasing to $\sim 50 \AA$ or the angle of LAGB increasing to $\sim 3^{\circ}$, the stress field radii of the dislocations for all planes are suppressed to be $\sim 40 \AA$. Based on the calculated results, it is reasonable to speculate that the HAGB and tilt LAGB with angle larger than $3^{\circ}$ can be studied from first-principles calculations, such as direct ab initio calculations [77, 85, 88, 89], due to their small stress fields.

To investigate the variation feature of the strain field, the disregistry for all tilt LAGBs is calculated and the results are shown in Fig. 3d-f. One can see that the line property does not change for all cases. With decreasing mean distance between dislocations in LAGBs or with increasing GB angle, the strain field ( 0 and 1 show no strains) is also gradually suppressed and the strain gradient is increased. The results agree with the kernel average misorientation (KAM) experimental findings that the strain fields of dislocations in small angle GBs are clearly larger than that in larger angle GBs [38]. The increased strain gradient enlarges the dilation/compression near the dislocation cores, and thus the size-interaction between solute atoms and tilt LAGB increases [50]. At equilibrium state, more solute atoms segregate to larger angle tilt GBs, which may promote the nucleation of precipitates. The strain field of prismatic dislocation has the largest variation with decreasing mean distance between dislocations in (1120) tilt LAGB, while those of basal and pyramidal dislocations in tilt LAGBs change comparably.

The grain boundary energy (GBE) is an important physical quantity that influences the recovery, strain hardening, recrystallization, and grain growth of materials during deformation or heat treatment. However, its accurate evolution behavior has not been clearly understood due to the difficulties in treatment of dislocation cores. To study the GBEs of tilt LAGBs, the dislocation line energies (DLEs) of

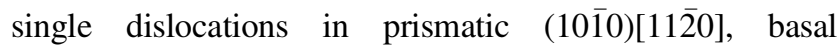

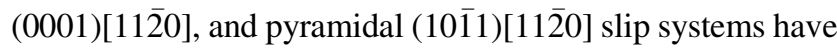
been calculated first. The results together with the values estimated from the continuous model $\left(E_{\mathrm{dis}}=\frac{K b}{4 \pi} \ln \frac{R}{2 \zeta}\right)$ are shown in Table 3. Other dislocation properties are also presented. It can be seen that the DLEs for all planes are about $1 \mathrm{eV} / \AA$ ( $(1.16669,1.18331$ and $1.25359 \mathrm{eV} / \AA$ for basal, prismatic, and pyramidal edge full $<\mathrm{a}>$ dislocations, respectively), in the same order as most experiment measurements [41]. The present dislocation line energies calculated from the discrete crystal model are slightly larger than those from continuous model. The dislocation on pyramidal plane holds the largest DLE, while that on the prismatic plane has the smallest one, which is consistent with that calculated from continuous model.

Based on the improved Peierls-Nabarro model, we calculated the DLE and GBE as well as the dislocation core energy in symmetric tilt LAGBs of Mg. The DLEs as a function of mean distance between dislocations and the GBEs plotted against tilt angles in LAGBs are shown in Fig. $4 \mathrm{a}$ and $\mathrm{b}$, respectively. For all cases, the DLE can be divided into three stages with decreasing mean distance between dislocations. Firstly, the DLE decreases slowly and nearly linearly with decreasing mean distance from the infinite value to $\sim 300 \AA$, and the reduction is about $10 \%$, implying that the interaction between dislocations is very small. Then the DLE declines exponentially and quickly until the distance decreases to about $100 \AA$. In this region, the strong interaction between dislocations may benefit the climb mechanism due to the $70 \%$ consumption of the

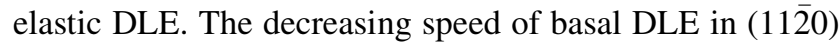
tilt LAGB is the smallest with decreasing mean distance between dislocations, while those of the prismatic and pyramidal DLEs possess the comparable and larger ones. The reason may be that the elastic energy factor $K$ of basal dislocation is the smallest (being $26.34 \mathrm{GPa}$ ), whereas the prismatic and pyramidal dislocations have comparable and larger values, being 27.32 and $27.65 \mathrm{GPa}$, respectively (see Table 3). The DLE changes small at the third stage, of which the total energy reduction is about $20 \%$. Since the mean distance between dislocations is about several times of Burgers vector, the DLE is closely related to the properties of the dislocation core. Dislocation with larger half width has the smaller DLE in this region owing to the easy compression of the dislocation crystal. Therefore, the pyramidal dislocation in tilt LAGBs has the largest DLE, followed by the basal dislocation and the prismatic dislocation possesses the smallest one. This feature of DLE is different from that derived from continuous model 

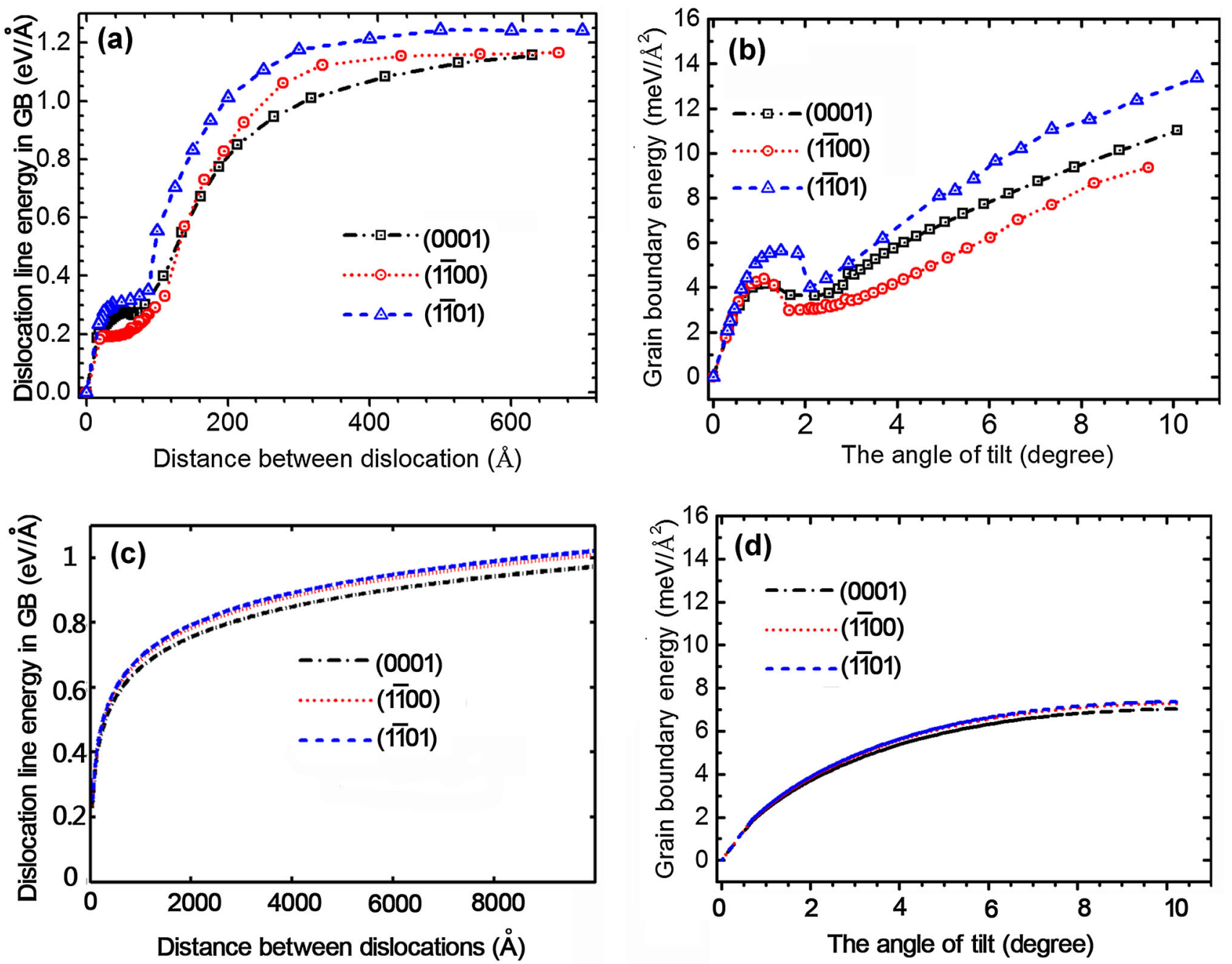

Fig. 4 a Dislocation line energies of basal, prismatic and pyramidal edge full $<$ a $>$ dislocations as a function of distance between dislocations in symmetric (1120) tilt LAGBs; b grain boundary energies plotted against tilt angles; c, $\mathbf{d}$ corresponding values estimated from conventional theory, respectively

$E_{\mathrm{LAGB}}=\frac{K b^{2}}{4 \pi D} \ln \frac{e \alpha D}{2 \pi b}[41]$ (see Fig. $4 \mathrm{c}$ ), where the DLE changes smoothly and slowly and $\alpha=1$ relative to the dislocation core effect. Since $\alpha$ cannot be determined by traditional elastic theory, the variation behavior of DLE in tilt LAGB is actually empirical.

The GBE variation property against tilt angle is shown in Fig. 4b. Previous theoretical prediction from $E_{\mathrm{LAGB}}^{\prime}=$ $\frac{K b}{4 \pi} \theta \ln \frac{e \alpha}{2 \pi \theta}[90]$, as shown in Fig. 4d, gives a total increase of GBE with increasing tilt angle. However, present work shows that the GBE increases very quickly at first until the angle is about $1^{\circ}$. Then the GBE slightly decreases when the tilt angle increases from $1^{\circ}$ to about $2^{\circ}, \sim 0.8 \mathrm{meV} / \AA^{2}$ for the tilt LAGB with array of (0001) basal dislocations, and $\sim 1.6 \mathrm{meV} / \AA^{2}$ for the rest, about twice that of the former. The difference maybe originated from the fact that the stress and strain fields in dislocation cores change when investigated by using Peierls-Nabarro model. Consequently, the coefficient $\alpha$ varies with increasing mean distance between the dislocations in tilt LAGBs. Finally, the GBE increases gradually with increasing angle of the tilt grain boundary. In the whole range, the tilt LAGB with array of (1101) pyramidal dislocations always has the largest GBE, while that consisting of $(1 \overline{1} 00)$ prismatic dislocations has the smallest one.

In order to investigate the effect of dislocation core on the energy variation of tilt LAGB, the misfit energies estimated from Eq. (13) as a function of distance between dislocations in tilt LAGBs of $\mathrm{Mg}$ were further calculated. The results are plotted in Fig. 5. One can see that there is a critical distance (about $400 \AA$ for all LAGBs), beyond which the core energy is nearly unchanged, indicating that the interaction between dislocation cores vanishes. Then the change of the core energy is separated into two stages with decreasing mean distance between the dislocations. At 


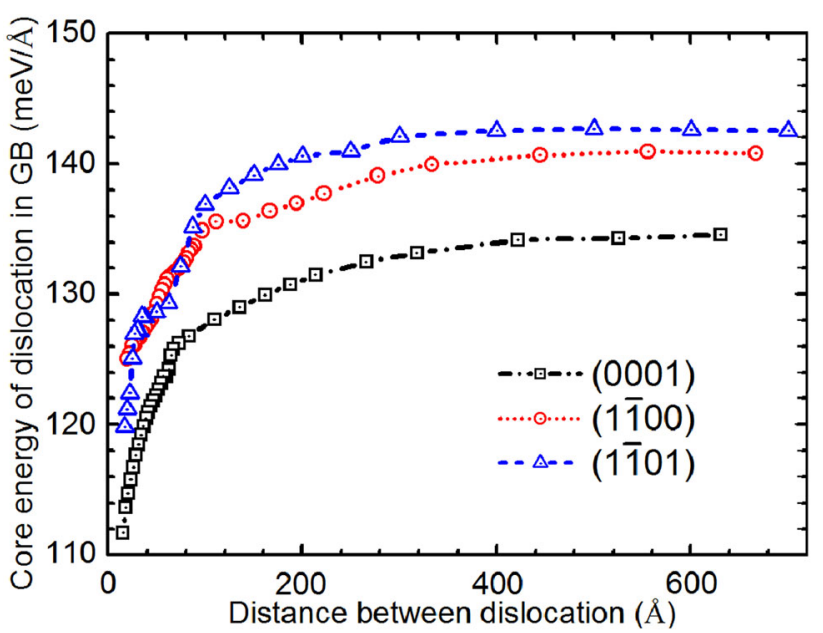

Fig. 5 Dislocation core energies as a function of distance between the dislocations in the $(11 \overline{2} 0)$ tilt LAGBs with array of prismatic, basal and pyramidal edge full $<\mathrm{a}>$ dislocations, respectively

the first stage, from the critical value to $\sim 100 \AA$, the core energy decreases slowly and nearly linearly. The reduction for all LAGBs is identical, $\sim 6 \mathrm{meV} / \AA$. The decreasing speed of the dislocation core energy increases at the second stage (from $\sim 100 \AA$ down to limit value), implying the strong interaction between dislocation cores. The change of core energy is also linear within this distance for all cases, $\sim 12 \mathrm{meV} / \AA$ for the $(11 \overline{2} 0)$ tilt LAGB with array of (1100) prismatic dislocations and $\sim 20 \mathrm{meV} / \AA$ for the other two. From Figs. 4, 5, it can be seen that for all LAGBs, the variation of dislocation core energy is rather small compared to that of DLE, resulting in larger ratio of the dislocation core energy to DLE. The core energy is not always $10 \%$ of DLE in grain boundary [91]. Especially at a

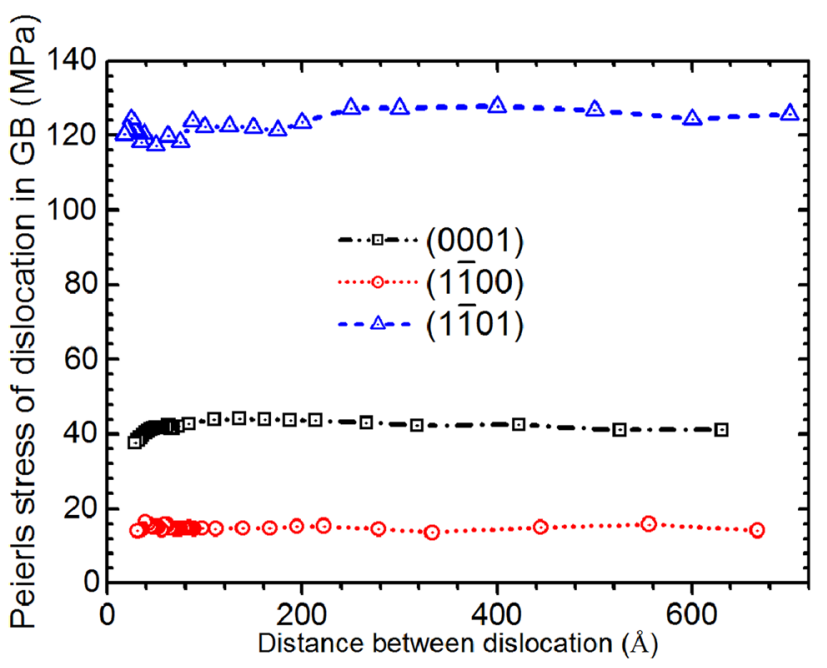

Fig. 6 Peierls stresses of basal, prismatic, and pyramidal dislocations in (1120) tilt LAGB as a function of distance between dislocations distance less than $100 \AA$, the core energy $(\sim 0.1 \mathrm{eV} / \AA)$ is nearly half of the DLE $(\sim 0.2 \mathrm{eV} / \AA)$ and its effect on GBE cannot be ignored.

Finally, the motion character is investigated through calculating the Peierls stress from Eq. (14), and the results are presented in Fig. 6. Although the stress field and DLE largely changes, the Peierls stress of dislocation in all GBs nearly remains unchanged, the same as those of single dislocations. The array of dislocations in LAGBs should not show strengthening effect if described by the collective movement of the individual straight dislocations at low temperatures [92]. This is in agreement with the trends in previous findings through molecular dynamics (MD) simulation [93] and other theoretical predictions [94]. With increasing temperature, the thermal activation should make the slipping of dislocation easier and the Peierls stress should be reduced [41, 95]. However, due to the interaction between dislocations in symmetric tilt LAGBs, the effect of thermal activation should be reduced. From this point of view, arrays of dislocations in LAGBs may also show strengthening effect at higher temperatures, for which additional experimental evidence is needed.

\section{Summary}

In summary, three types of symmetric (1120) tilt low-angle grain boundaries (LAGBs) with array of basal, prismatic, and pyramidal edge full $<\mathrm{a}>$ dislocations in pure $\mathrm{Mg}$ were studied. The basic stress equation for tilt LAGBs was taken from the Peierls-Nabarro model and minimized with the restoring force derived from GSFE curve to study the properties of tilt LAGBs. The results show that with decreasing distance between the dislocations in tilt LAGBs, the stress and strain fields are gradually suppressed. The variation degree of prismatic, basal, and pyramidal edge full $<\mathrm{a}>$ dislocations in tilt LAGBs decreases sequentially. As the distance between the dislocations decreasing to $\sim 50 \AA$ or the angle of GB increasing to $\sim 3^{\circ}$, the radius of the dislocation stress field in all GBs fall to $\sim 40 \AA$. The dislocation line energy (DLE) is divided into three stages with decreasing mean distance between dislocations in all tilt LAGBs. The DLE changes slightly and linearly, $\sim 10 \%$, when the distance is larger than $300 \AA$ and then declines very quickly from 300 to $100 \AA, \sim 70 \%$. Finally, the decreasing speed is lowered and the dislocation core effect cannot be neglected in this region. The core energy is nearly half of the DLE. The grain boundary energy (GBE) decreases when the tilt angle of LAGB increases from $1^{\circ}$ to $2^{\circ}$. The $(11 \overline{2} 0)$ tilt LAGB with array of $(1 \overline{101})$ pyramidal dislocations always has the largest GBE, while that consisting of (1100) prismatic dislocations has the smallest one 
in the whole range. The Peierls stress of dislocation in all GBs is nearly not changed, the same as that of the single dislocation, indicating that arrays of dislocations in LAGBs would not show a strengthening effect at low temperatures.

Acknowledgments This work is supported by the National Natural Science Foundation of China (Nos. 11427806, 51471067, 51371081, 51171063,51501059 and 51501060), the National Basic Research (973) Program of China (No. 2009CB623704), the Chinese Postdoctoral Science Foundation (No. 2015M582324), and the Hunan Provincial Natural Science Foundation (No. 14JJ4052) and the Science and Technology Project for Good Postdoctoral Education of China (No. 2015RS4020) .

\section{References}

[1] T.M. Pollock, Science 328, 986 (2010)

[2] X.H. Chen, L.Z. Liu, J. Liu, F.S. Pan, Acta Metall. Sin. (Engl. Lett.) 28, 492 (2015)

[3] C. Potzies, K.U. Kainer, Adv. Eng. Mater. 6, 281 (2004)

[4] R. Gehrmann, M.M. Frommert, G. Gottstein, Mater. Sci. Eng., A 395, 338 (2005)

[5] C.H. Cáceres, A. Blake, Phys. Stat. Sol. (A) 194, 147 (2002)

[6] V.M. Segal, Mater. Sci. Eng., A 271, 322 (1999)

[7] Y. Wang, M. Chen, F. Zhou, E. Ma, Nature 419, 912 (2002)

[8] Y.T. Zhu, X. Liao, Nat. Mater. 3, 351 (2004)

[9] L. Wang, E. Mostaed, X. Cao, G. Huang, A. Fabrizi, F. Bonollo, C. Chi, M. Vedani, Mater. Des. 89, 1 (2016)

[10] J. Zhang, Z. Kang, L. Zhou, Mater. Sci. Eng., A 647, 184 (2015)

[11] Y. Yuan, A. Ma, X. Gou, J. Jiang, F. Lu, D. Song, Y. Zhu, Mater. Sci. Eng., A 630, 45 (2015)

[12] H.G. Svoboda, F. Vago, Proc. Mater. Sci. 9, 590 (2015)

[13] E. Mostaed, A. Fabrizi, D. Dellasega, F. Bonollo, M. Vedani, J. Alloys Compd. 638, 267 (2015)

[14] P. Minárik, R. Král, J. Pešička, F. Chmelík, J. Mater. Res. Technol. 4, 75 (2015)

[15] M. Gzyl, A. Rosochowski, S. Boczkal, L. Olejnik, Mater. Sci. Eng., A 638, 20 (2015)

[16] J. Zhang, C. Xin, K. Nie, W. Cheng, H. Wang, C. Xu, Mater. Sci. Eng., A 611, 108 (2014)

[17] P.J. Hsieh, Y.P. Hung, J.C. Huang, Scripta Mater. 49, 173 (2003)

[18] G. Garces, M.A. Muñoz-Morris, D.G. Morris, P. Perez, P. Adeva, Mater. Sci. Eng., A 614, 96 (2014)

[19] R. Jahadi, M. Sedighi, H. Jahed, Mater. Sci. Eng., A 593, 178 (2014)

[20] X. Sauvage, F. Wetscher, P. Pareige, Acta Mater. 53, 2127 (2005)

[21] F. Li, W. Shi, N. Bian, H.B. Wu, Acta Metall. Sin. (Engl. Lett.) 28, 649 (2015)

[22] L. Dupuy, J.J. Blandin, Acta Mater. 50, 3253 (2002)

[23] Z. Horita, T. Fujinami, M. Nemoto, T.G. Langdon, J. Mater. Process. Technol. 117, 288 (2001)

[24] A. Hassani, M. Zabihi, Mater. Des. 39, 140 (2012)

[25] R.Z. Valiev, E.V. Kozlov, Y.F. Ivanov, J. Lian, A.A. Nazarov, B. Baudelet, Acta Metal. Mater. 42, 2467 (1994)

[26] Y.M. Wang, E. Ma, Acta Mater. 52, 1699 (2004)

[27] K.V. Ivanov, E.V. Naydenkin, Mater. Sci. Eng., A 608, 123 (2014)

[28] W.J. Kim, S.I. Hong, Y.S. Kim, S.H. Min, H.T. Jeong, J.D. Lee, Acta Mater. 51, 3293 (2003)

[29] M. Mabuchi, H. Iwasaki, K. Yanase, K. Higashi, Scripta Mater. 36, 681 (1997)
[30] T.C. Chang, J.Y. Wang, C.L. Chu, S. Lee, Mater. Lett. 60, 3272 (2006)

[31] A. Yamashita, Z. Horita, T.G. Langdon, Mater. Sci. Eng., A 300, $142(2001)$

[32] R.Z. Valiev, I.V. Alexandrov, Y.T. Zhu, T.C. Lowe, J. Mater. Res. 17, 5 (2002)

[33] R. Valiev, Nat. Mater. 3, 511 (2004)

[34] Y.H. Zhao, J.F. Bingert, Y.T. Zhu, X.Z. Liao, R.Z. Valiev, Z. Horita, T.G. Langdon, Y.Z. Zhou, E.J. Lavernia, Appl. Phys. Lett. 92, 081903 (2008)

[35] S. Biswas, S. Singh Dhinwal, S. Suwas, Acta Mater. 58, 3247 (2010)

[36] R.Z. Valiev, R.K. Islamgaliev, I.V. Alexandrov, Prog. Mater Sci. 45, 103 (2000)

[37] O.S. Sitdikov, R.O. Kaybyshev, I.M. Safarov, I.A. Mazurina, Phys. Met. Metallogr. 92, 270 (2001)

[38] P. Molnár, A. Jäger, P. Lejček, J. Mater. Sci. 47, 3265 (2012)

[39] A.P. Sutton, R.W. Balluffi, Interfaces in Crystalline Materials (Oxford University Press, New York, 1995)

[40] M. Peach, J.S. Koehler, Phys. Rev. 80, 436 (1950)

[41] J.P. Hirth, J. Lothe, Theory of Dislocations, 2nd edn. (Wiley, New York, 1982)

[42] I.A. Ovid'ko, A.G. Sheinerman, R.Z. Valiev, Scripta Mater. 76, 45 (2014)

[43] J.D. Eshelby, W.T. Read, W. Shockley, Acta Metall. 1, 251 (1953)

[44] J.E. Lennard-Jones, Proc. Phys. Soc. 43, 461 (1931)

[45] D.Y. Sun, M.I. Mendelev, C.A. Becker, K. Kudin, T. Haxhimali, M. Asta, J.J. Hoyt, A. Karma, D.J. Srolovitz, Phys. Rev. B 73, 024116 (2006)

[46] X.Y. Liu, J.B. Adams, F. Ercolessi, J.A. Moriarty, Model. Simul. Mater. Sci. Eng. 4, 293 (1996)

[47] V. Vitek, M. Igarashi, Philos. Mag. A 63, 1059 (1991)

[48] J.A. Yasi, T. Nogaret, D.R. Trinkle, Y. Qi, L.G. Hector Jr., W.A. Curtin, Model. Simul. Mater. Sci. Eng. 17, 055012 (2009)

[49] Y. Tang, J.A. El-Awady, Acta Mater. 71, 319 (2014)

[50] T.W. Fan, L.G. Luo, L. Ma, B.Y. Tang, L.M. Peng, W.J. Ding, Mater. Sci. Eng., A 582, 299 (2013)

[51] B. Joós, Q. Ren, M.S. Duesbery, Phys. Rev. B 50, 5890 (1994)

[52] J. Hartford, B. von Sydow, G. Wahnström, B.I. Lundqvist, Phys. Rev. B 58, 2487 (1998)

[53] G. Schoeck, Acta Mater. 49, 1179 (2001)

[54] P. Carrez, D. Ferré, P. Cordier, Nature 446, 68 (2007)

[55] T.W. Fan, Q. Zhang, L. Ma, P.Y. Tang, B.Y. Tang, L.M. Peng, W.J. Ding, Eur. J. Mech. A-Solids 45, 1 (2014)

[56] S. Kibey, J.B. Liu, D.D. Johnson, H. Sehitoglu, Acta Mater. 55, 6843 (2007)

[57] S. Kibey, J.B. Liu, M.J. Curtis, D.D. Johnson, H. Sehitoglu, Acta Mater. 54, 2991 (2006)

[58] G. Lu, N. Kioussis, V.V. Bulatov, E. Kaxiras, Phil. Mag. Lett. 80, 675 (2000)

[59] G. Schoeck, Philos. Mag. A 69, 1085 (1994)

[60] G. Schoeck, M. Krystian, Philos. Mag. 85, 949 (2005)

[61] G.P.M. Leyson, W.A. Curtin, Philos. Mag. 93, 2428 (2013)

[62] V. Vitek, Philos. Mag. 18, 773 (1968)

[63] S. Dai, Y. Xiang, D.J. Srolovitz, Acta Mater. 61, 1327 (2013)

[64] S. Dai, Y. Xiang, D.J. Srolovitz, Acta Mater. 69, 162 (2014)

[65] W.W. Hu, Z.Q. Yang, H.Q. Ye, Scripta Mater. 117, 77 (2016)

[66] G. Kresse, J. Hafner, Phys. Rev. B 47, 558 (1993)

[67] G. Kresse, J. Furthmüller, Comp. Mater. Sci. 6, 15 (1996)

[68] G. Kresse, J. Furthmüller, Phys. Rev. B 54, 11169 (1996)

[69] P.E. Blöchl, Phys. Rev. B 50, 17953 (1994)

[70] J.P. Perdew, K. Burke, M. Ernzerhof, Phys. Rev. Lett. 77, 3865 (1996)

[71] H.J. Monkhorst, J.D. Pack, Phys. Rev. B 13, 5188 (1976) 
[72] J.A. Yan, C.Y. Wang, S.Y. Wang, Phys. Rev. B 70, 174105 (2004)

[73] B. Joós, M. Duesbery, Phys. Rev. Lett. 78, 266 (1997)

[74] V.V. Bulatov, E. Kaxiras, Phys. Rev. Lett. 78, 4221 (1997)

[75] Z. Pei, L.F. Zhu, M. Friák, S. Sandlöbes, J.V. Pezold, H.W. Sheng, C.P. Race, S. Zaefferer, B. Svendsen, D. Raabe, J. Neugebauer, N. J. Phys. 15, 043020 (2013)

[76] M. Muzyk, Z. Pakiela, K.J. Kurzydlowski, Scripta Mater. 66, 219 (2012)

[77] I. Shin, E.A. Carter, Model. Simul. Mater. Sci. Eng. 20, 015006 (2012)

[78] T. Nogaret, W.A. Curtin, J.A. Yasi, L.G. Hector Jr., D.R. Trinkle, Acta Mater. 58, 4332 (2010)

[79] X. Wu, R. Wang, S. Wang, Appl. Surf. Sci. 256, 3409 (2010)

[80] Q. Zu, Y.F. Guo, X.Z. Tang, Acta Metall. Sin. (Engl. Lett.) 28, 876 (2015)

[81] J. Han, X.M. Su, Z.H. Jin, Y.T. Zhu, Scripta Mater. 64, 693 (2011)

[82] T. Uesugi, M. Kohyama, M. Kohzu, K. Higashi, Mater. Sci. Forum 419-422, 225 (2003)
[83] J.C.M. Li, Acta Metall. 8, 296 (1960)

[84] J.C.M. Li, Acta Metall. 8, 563 (1960)

[85] J.A. Yasi, L.G. Hector Jr., D.R. Trinkle, Acta Mater. 58, 5704 (2010)

[86] Z.R. Liu, D.Y. Li, Acta Mater. 89, 225 (2015)

[87] S.H. Wang, C.H. Liu, J.H. Chen, X.L. Li, D.H. Zhu, G.H. Tao, Mater. Sci. Eng., A 585, 233 (2013)

[88] J.A. Yasi, L.G. Hector Jr., D.R. Trinkle, Acta Mater. 60, 2350 (2012)

[89] J.A. Yasi, L.G. Hector Jr., D.R. Trinkle, Acta Mater. 59, 5652 (2011)

[90] W.T. Read, W. Shockley, Phys. Rev. 78, 275 (1950)

[91] J.P. Hirth, Metall. Trans. 3, 3047 (1972)

[92] T.J. Rupert, D.S. Gianola, Y. Gan, K.J. Hemker, Science 326, 1686 (2009)

[93] A.T. Lim, Mechanical and Aerospace Engineering (Princeton University, Candidacy, 2012)

[94] T. Vreeland Jr., Acta Metall. 7, 240 (1959)

[95] A. Akhtar, E. Teghtsoonian, Acta Metall. 17, 1339 (1969) 